\title{
Combined Electroejaculation and in Vitro Fertilization in the Evaluation and Treatment of Anejaculatory Infertility
}

\author{
JOHN F. RANDOLPH, JR., ${ }^{1,2}$ DANA A. OHL,${ }^{3}$ CAROL J. BENNETT, ${ }^{4}$ JONATHAN W. T. AYERS, ${ }^{5}$ \\ and ALAN C. MENGE
}

Submitted: August 25, 1989

Accepted: December 29, 1989

\begin{abstract}
Seven couples underwent combined electroejaculation and in vitro fertilization for anejaculatory infertility after a failed regimen of electroejaculation and intrauterine insemination. Two pregnancies resulted, one proceeding to a term vaginal delivery and one ending in a 6-week spontaneous abortion. Poor sperm binding and no fertilization were seen in two of the couples. Fertilization of the oocytes but no subsequent pregnancy were seen in the other three couples. Combined in vitro fertilization and gamete intrafallopian transfer was performed in four of the couples. The combination of electroejaculation and in vitro fertilization offers the opportunity to evaluate the female pelvis, observe the sperm-oocyte interaction, and achieve a pregnancy in couples with anejaculatory infertility.
\end{abstract}

KEY WORDS: electroejaculation; anejaculation; in vitro fertilization.

\section{INTRODUCTION}

Male-factor infertility as a result of anejaculation may occur following spinal cord injury or retroperitoneal surgery or as a result of multiple sclerosis, diabetes mellitus, or psychogenic causes. Electro-

\footnotetext{
${ }^{1}$ Division of Reproductive Endocrinology, Department of $\mathrm{Ob} /$ Gyn, University of Michigan Medical Center, Ann Arbor, Michigan 48109.

2 To whom correspondence should be addressed at Department Ob/Gyn, Box 0718, University of Michigan Medical Center, 1500 East Medical Center Drive, Ann Arbor, Michigan 481090718 .

${ }^{3}$ Section of Urology, Department of Surgery, University of Michigan Medical Center, Ann Arbor, Michigan 48109.

${ }^{4}$ Department of Urology, University of Southern California, Los Angeles, California 90024.

${ }^{5}$ St. Joseph's Mercy Hospital, Ann Arbor, Michigan.
}

ejaculation facilitates the collection of sperm, permitting processing and insemination and resulting in successful pregnancies (1) in this previously infertile group of patients.

Two critical factors in the success of electroejaculation/insemination are the evaluation of the female partner and subsequent timing of inseminations. The evaluation of the female ultimately includes a diagnostic laparoscopy to rule out pelvic factors for infertility. In addition to visualizing the pelvis, laparoscopy provides an opportunity for oocyte retrieval, gamete intrafallopian transfer (GIFT), and in vitro fertilization (IVF) to assess the capacity of sperm obtained by electroejaculation to penetrate the spouse's oocytes. This paper presents the first seven couples undergoing combined electroejaculation and IVF/GIFT in the Electroejaculation/Insemination Program at the University of Michigan from December 1986 through November 1988.

\section{MATERIALS AND METHODS}

Patients. Anejaculatory males were referred to the Section of Urology, Department of Surgery, at the University of Michigan Medical Center for evaluation and possible electroejaculation. The cause of anejaculation was spinal cord injury in six men and radical retroperitoneal lymph node dissection (RPLND) for testicular carcinoma in the remaining patient. The period of anejaculation ranged from 2 to 19 years.

After preliminary male evaluation, all female partners were evaluated by the Division of Reproductive Endocrinology and Infertility, Department of Obstetrics and Gynecology, at the University of 
Michigan Medical Center. All were in good health without significant medical problems. One patient was previously noted to have a normal pelvis at the time of diagnostic laparoscopy to rule out suspected endometriosis. A second patient had undergone a right salpingostomy for an ampullary ectopic pregnancy but demonstrated bilateral tubal patency on subsequent hysterosalpingography. None of the other patients had any risk factors for tubal infertility.

The males ranged from 28 to 37 years of age, whereas the females ranged from 24 to 37 years of age at the time of the stimulation cycle. Four of the patients were nulligravidas, with only patient 6 having successfully carried a child in the past.

Program Protocol. The Electroejaculation/ Insemination Program is divided into an initial evaluation phase, a second insemination phase, and a third IVF phase. The initial phase consists of the evaluation of the male and a variable number of preliminary electroejaculation procedures to establish the optimal stimulation and appropriate response. The female partner was evaluated just prior to entry into the second phase of the Program.

Male partners received $1.5 \mathrm{~g}$ sodium bicarbonate orally at $12 \mathrm{AM}$ on the night prior to electroejaculation and again $1 \mathrm{hr}$ before the procedure to achieve a urine $\mathrm{pH}$ of $7-8$ at the time of the procedure. If the patient had a high spinal cord injury, and it was deemed autonomic dysreflexia would be a risk, 20 $\mathrm{mg}$ sublingual nifedipine was given for prophylaxis. Patients with incomplete spinal cord lesions were given midazolam for sedation. The RPLND patient had normal sensation and required general anesthesia for the electroejaculation procedure. Immediately preceding electroejaculation a catheterized urine specimen was tested for acidity and the bladder was washed with intrauterine insemination (IUI) medium [Hamm's F-10 (Gibco No. 430-1200), Hepes buffer, $20 \mathrm{mM}$ (Sigma No. H3375), and 1\% human serum albumin (Fraction V, 96-99\% pure, Cutter 685-20)] if the $\mathrm{pH}$ was less than 7.0. A Model 10 electroejaculator probe ( $\mathrm{G}$ and $\mathrm{S}$ Instrument Co., M-10) was inserted 6-8 in. into the anal canal with the patient in the lateral decubitus position. Progressively increasing voltage was delivered in a wavelike pattern until ejaculation was achieved. Stimulation was initiated at 2-3 V, with each subsequent stimulation occurring $6-8 \mathrm{sec}$ later with an increase in voltage of $1 \mathrm{~V}$. Blood pressure was monitored throughout the procedure, which utilized an average of $19 \mathrm{~V}$ and $450 \mathrm{amp}$. The antegrade sperm were collected by milking the phallus. After the procedure the bladder was catheterized to collect the retrograde ejaculate. Five to ten $\mathrm{ml}$ of IUI medium was utilized to flush the bladder to obtain any residual ejaculate.

Both antegrade and retrograde samples were washed with IUI medium, followed by a "swimup" for $1 \mathrm{hr}$. The supernatant containing motile spermatozoa was removed and centrifuged for 6 min at $400 \mathrm{~g}$. The pellet was resuspended in IUI medium and a count, percentage motility, and forward progression were determined. A second centrifugation was performed, with the resulting pellet resuspended in $250 \mu \mathrm{l}$ of IUI medium. The second sample in couple 2 was swum-up from IUI medium containing 5\% human serum albumin and all three oocytes collectively inseminated with $5 \times 10^{5}$ sperm.

The evaluation of the female partner included an initial history and physical examination, the recording of basal body temperatures, and a hysterosalpingogram to document bilateral tubal patency. For the insemination phase of the program, all female patients were placed on clomiphene citrate, $50 \mathrm{mg} /$ day, on days 5-9 of each cycle to assure predictable timing for subsequent electroejaculation/insemination procedures. Patients were seen on the day before expected ovulation, at which time transvaginal ultrasonography was performed to document the presence of a preovulatory follicle. Human chorionic gonadotropin ( $\mathrm{hCG}$ ), 10,000 $\mathrm{U}$, was administered im if the follicle was greater than or equal to $17 \mathrm{~mm}$ in mean diameter. The male partner underwent electroejaculation on the day of hCG and on the following day, with subsequent sperm processing and intrauterine insemination each day.

All couples had undergone at least six cycles of electroejaculation/insemination without pregnancy before proceeding to the third phase of the program. One couple had undergone five of six attempts at a different program. The first four women underwent a superovulation protocol consisting of $225 \mathrm{U}$ of human menopausal gonadotropins (hMG) daily beginning on day 3 of the menstrual cycle. Vaginal ultrasonography and serum testing for estradiol and progesterone concentrations were begun on day 5 of the cycle, with repeat studies every $24-48 \mathrm{hr}$. The last three patients in the series began leuprolide on day 21 of the cycle preceding the IVF cycle and began $225 \mathrm{U}$ of hMG daily 10 days after beginning leuprolide therapy. Monitoring of these patients was identical to that of the first four pa- 
tients in the series. When at least two follicles were greater than or equal to $16 \mathrm{~mm}$ in mean diameter and the estradiol level was greater than or equal to $500 \mathrm{pg} / \mathrm{ml}, 10,000 \mathrm{U}$ hCG was administered im and the laparoscopy scheduled for $36 \mathrm{hr}$ later.

Electroejaculation was performed several hours prior to scheduled laparoscopy to allow for sperm processing. Laparoscopy was carried out under general anesthesia, with all patients demonstrating normal anatomy. Follicular aspiration was performed in a standard fashion and GIFT performed with a maximum of four oocytes if the processed sperm were deemed appropriate. The GIFT procedures utilized a Marrs catheter (Cook Ob/Gyn, Spencer, IN) loaded with $5 \mu$ l medium, $1.25 \times 10^{5}$ sperm in $25 \mu$ l medium, $1-2$ oocytes in $20 \mu \mathrm{l}$ medium containing $7.5 \%$ female serum, $1 \mu 1$ air, and 1 $\mu \mathrm{l}$ medium. Oocytes were equilibrated and inseminated with $1.0 \times 10^{5}$ to $2.5 \times 10^{5}$ sperm per dish 4-6 $\mathrm{hr}$ after retrieval. Sperm binding was assessed later that day and, in one patient, deemed to be unacceptable; therefore, a second ejaculation was performed that day with reinsemination of the oocytes. The oocytes were evaluated the following morning and repeat ejaculation and reinsemination were performed if fertilization failed as in two of the patients. Embryo transfer was carried out $44-48 \mathrm{hr}$ after oocyte retrieval. Patients on the hMG stimu- lation protocol received progesterone suppositories, $100 \mathrm{mg}$ bid, beginning on the day after embryo transfer, whereas the patients receiving leuprolide/ hMG began progesterone, $25 \mathrm{mg}$ im daily, on the day following oocyte retrieval. Two weeks after oocyte retrieval a quantitative serum beta hCG level was obtained. If it was positive, the patient was maintained on progesterone; if it was negative, progesterone was discontinued and menses ensued.

\section{RESULTS}

The results are listed in Table I. Two of the seven couples conceived, with one pregnancy progressing to a term singleton delivery and the second ending in a spontaneous abortion at 6 weeks of gestation as evidenced by the passage of gestational tissue. Chromosomal analysis of the aborted tissue was not obtained. The number of eggs obtained ranged from 3 to 12 , with all eggs in excess of 6 frozen for possible future use.

Initial sperm counts ranged from $0.1 \times 10^{6}$ to 40 $\times 10^{6} / \mathrm{ml}$, with a range of motility from 1 to $75 \%$. Sperm binding and fertilization were noted in five of the seven couples, with all of the couples demonstrating some progression of fertilized oocytes to cell cleavage.

Table I. Couples Undergoing IVF/GIFT After Electroejaculation, December 1986 Through November 1988

\begin{tabular}{|c|c|c|c|c|c|c|c|c|c|c|c|c|c|c|c|}
\hline $\begin{array}{l}\text { Pt } \\
\text { No. }\end{array}$ & $\begin{array}{c}\text { Female } \\
\text { age }\end{array}$ & G-P & $\mathrm{Rx}$ & $\begin{array}{c}\text { Peak } \\
E_{2}\end{array}$ & $\begin{array}{l}\text { day } \\
\text { hCG }\end{array}$ & $\begin{array}{c}\text { No. } \\
\text { eggs } \\
\text { (frozen) }\end{array}$ & GIFT & $\begin{array}{c}\text { No. } \\
\text { fertil- } \\
\text { ized }\end{array}$ & $\begin{array}{l}\text { No. } \\
\text { trans- } \\
\text { ferred }\end{array}$ & $\begin{array}{l}\text { Out- } \\
\text { come }\end{array}$ & $\begin{array}{c}\text { Male } \\
\text { age }\end{array}$ & Level & $\begin{array}{c}\text { Time } \\
\text { (years) }\end{array}$ & $\begin{array}{l}\text { Count } \\
\left(\times 10^{6}\right)\end{array}$ & $\begin{array}{c}\% \\
\text { motil } \\
\text { ity }\end{array}$ \\
\hline 1 & 24 & G1P0 & hMG & 1089 & 10 & 8 & $\begin{array}{l}\text { Yes } \\
1(\mathrm{R}) \\
2(\mathrm{~L})\end{array}$ & 0 & 0 & $(-)$ & 29 & T6 & 2 & 40.0 & 45 \\
\hline 2 & 25 & G0 & hMG & 965 & 8 & 3 & No & 3 & 3 & $(+)^{a}$ & 28 & T12-L1 & 4 & $\begin{array}{l}\text { (1) } 0.1 \\
\text { (2) } 1.0\end{array}$ & $\begin{array}{c}1 \\
25-30\end{array}$ \\
\hline 3 & 36 & G0 & hMG & 1565 & 7 & $8(2)$ & $\begin{array}{l}\text { Yes } \\
2(\mathrm{R}) \\
2(\mathrm{~L})\end{array}$ & 2 & 2 & $(-)$ & 37 & C5-C6 & 19 & 10.0 & 50 \\
\hline 4 & 31 & G0 & hMG & 545 & 9 & 6 & $\begin{array}{l}\text { Yes } \\
2(\mathrm{R}) \\
1(\mathrm{~L})\end{array}$ & 2 & 2 & $(+)^{b}$ & 32 & T10-T11 & 5 & 6.0 & 80 \\
\hline 5 & 37 & G0 & $\begin{array}{c}\text { Lupron/ } \\
\text { hMG }\end{array}$ & 1584 & 13 & $9(3)$ & No & 0 & 0 & $(-)$ & 37 & $\mathrm{~T} 7$ & 14 & $\begin{array}{l}\text { (1) } 1.4 \\
\text { (2) } 0.2\end{array}$ & $\begin{array}{c}20-25 \\
25\end{array}$ \\
\hline 6 & 35 & $\mathrm{G} 2 \mathrm{P} 1$ & $\begin{array}{c}\text { Lupron } \\
\text { hMG }\end{array}$ & 1857 & 12 & $12(6)$ & $\begin{array}{l}\text { Yes } \\
2(\mathrm{R}) \\
2(\mathrm{~L})\end{array}$ & 2 & 2 & $(-)$ & 31 & RPLND & 4 & 14 & 75 \\
\hline 7 & 29 & G1P0 & $\underset{\text { hMG }}{\text { Lupron/ }}$ & 2163 & 13 & $8(2)$ & No & 5 & 5 & $(-)$ & 33 & T5-T6 & 5 & $\begin{array}{l}\text { (1) } 8.0 \\
\text { (2) } 0.6\end{array}$ & $\begin{array}{r}5 \\
65\end{array}$ \\
\hline
\end{tabular}

a Term birth.

${ }^{b}$ Six-week SAb. 
No fertilization was seen in two of the seven couples. In Couple 1, despite an initial count of $40 \times 10^{6}$ sperm $/ \mathrm{ml}$ with $45 \%$ motility, no binding of sperm to the oocyte was seen. Subsequent testing failed to reveal the presence of antisperm antibodies or mouse embryo toxicity in the female's serum. In Couple 5, poor sperm counts and motility were noted in two ejaculates with minimal binding and no fertilization. Couple 2 demonstrated a very poor count and motility in the sample obtained on the day of retrieval with an improved but still abnormal sample obtained the following day. The second sample successfully fertilized all three oocytes obtained and the subsequent embryo transfer resulted in a term singleton delivery.

\section{DISCUSSION}

Couples with anejaculatory infertility present a frustrating subgroup of patients with male-factor infertility. The effect of extended anejaculation on sperm production and function has not yet been well characterized. Moreover, the problem causing the anejaculation may result in other complications directly or indirectly affecting sperm quality such as chronic urinary tract infections in patients with spinal cord injuries.

Electroejaculation is now a clinically acceptable method for obtaining semen from men with chronic anejaculation (2). Since the first reports of successful conception with sperm obtained by electroejaculation $(3,4)$, the combination of electroejaculation and intrauterine insemination has resulted in pregnancies in couples with the male having suffered a spinal cord injury (1), with multiple sclerosis (5), after a retroperitoneal lymph node dissection for testicular carcinoma (6), and with psychogenic anejaculation (7).

A critical part of the success with electroejaculation has involved an appropriate evaluation of the female partner, appropriate timing of the electroejaculation and insemination, and the use of intrauterine insemination of processed sperm. In the Electroejaculation/Insemination Program at the University of Michigan, all female partners were evaluated for risk factors for infertility, for tubal patency, and for regular ovulation. Due to the distances many couples traveled to participate in the program and ensure predictable follicular development, all women were placed on clomiphene citrate and received hCG to assure ovulation at a time ap- propriate for insemination. Since all of the women had bilateral tubal patency and no other major risk factors for tubal infertility, a surgical evaluation was deferred until after a trial of electroejaculation and intrauterine insemination. When it was felt that conception had not occurred despite an adequate trial, the couples were offered the opportunity for IVF at the time of recommended laparoscopy. It was felt that little if any morbidity would be added to the procedure, that the quality of the sperm obtained at electroejaculation could be better assessed, and that a pregnancy might ensue. The use of IVF for male-factor infertility has been well described (8) and offers a viable alternative to couples with poor sperm numbers or quality. In some couples, the presence or absence of in vitro fertilization of the woman's oocytes by the man's sperm may be the deciding factor in determining whether to pursue other methods with which to become pregnant, such as donor insemination, or whether to pursue adoption or a childless life-style.

Only one of the seven female partners had carried a child to term before, with four of the women being nulligravid at the time of the IVF cycle. Both pregnancies established were in previously nulligravid patients.

Over the 2-year span of the series, the IVF protocol was modified. During the course of this series, embryo freezing was not available, although oocyte freezing was routinely performed rather than discarding excess oocytes that were not inseminated. Patient 3 had two oocytes frozen, with one ultimately surviving the freezing process, becoming fertilized, and being transferred to the woman in an unstimulated cycle without a subsequent conception.

Final sperm counts and motility varied widely among patients and were not reliable indicators of eventual fertilization. The highest count seen was in the couple where sperm binding was poor and no fertilization was noted. In contrast, the only term pregnancy reported resulted from a sample with a relatively low sperm concentration and decreased motility obtained in a repeat ejaculation the day following laparoscopy and retrieval (9). IVF and GIFT were performed in four of the seven couples due to the reported improved pregnancy rates with GIFT but the need to observe the in vitro sperm-egg interaction. Of note, all of the women demonstrated apparently normal pelves.

The number of patients in this series is too small to establish reliable success rates with this com- 
bined technique, but the occurrence of two pregnancies with one term delivery certainly demonstrates the potential of the technique. By demonstrating the fertilization of retrieved human oocytes, the technique provides a valuable in vitro assessment of the functional capacity of sperm obtained by electroejaculation.

\section{CONCLUSIONS}

In summary, this report describes the initial experience with electroejaculation and in vitro fertilization in an anejaculatory subset of couples with male-factor infertility and establishes this combined technique as a viable approach to the treatment of couples with a particularly frustrating type of infertility.

\section{ACKNOWLEDGMENT}

This work was supported in part by a grant from the Eastern Paralyzed Veterans of America.

\section{REFERENCES}

1. Bennett CJ, Ayers JWT, Randolph JF, et al.: Electroejaculation of paraplegic males followed by pregnancies. Fertil Steril 1987;48:1070-1072

2. Brindley GS: Electroejaculation: its technique, neurological implications and uses. J Neurol Neurosurg Psychiatry 1987;44:9

3. Thomas RJS, McLeish G, McDonald IA: Electroejaculation of the paraplegic male followed by pregnancy. Med J Aust 1975;2:798

4. Francois N, Maury M, Jovannet D, David G, Vacant J: Electroejaculation of a complete paraplegic followed by pregnancy. Para 1978;16:248

5. Ohl DA, Grainger R, Bennett CJ, Randolph JF, Seager SWJ, McCabe M: Successful use of electroejaculation in two anejaculatory multiple sclerosis patients including report of a pregnancy utilizing intrauterine insemination. Neurol Urodynam 1989;8:195-198

6. Bennett CJ, Ohl DA, McCabe M, Randolph J, Menge A: Electro-ejaculation in patients following retroperitoneal lymph node dissection. J Urol 1989;141(4):485A (abstr)

7. Stewart DE, Ohl DA: Idiopathic anejaculation treated by electroejaculation. Intl J Psychiat Med 1989;19(3)263-268

8. Acosta A, Kruger T, Swanson RJ, et al.: The role of in vitro fertilization in male infertility. Ann NY Acad Sci 1988;541: 297

9. Ayers JWT, Moinipanah BS, Bennett CJ, Randolph JF, Peterson EP: Successful combination therapy with electroejaculation and in vitro fertilization-embryo transfer in the treatment of a paraplegic male with severe oligoasthenospermia. Fertil Steril 1988;49(6):4194 